\title{
Eficácia do canabidiol na melhora da qualidade de vida do paciente com Parkinson: revisão integrativa
}

\author{
Efficacy of cannabidiol in improving the quality of life with Parkinson's Patient: integrative review \\ Eficacia del cannabidiol para mejorar la calidad de vida de los pacientes de Parkinson: una
} revisión integradora

Recebido: 18/09/2021 | Revisado: 26/09/2021 | Aceito: 30/09/2021 | Publicado: 03/10/2021

\author{
Marianna Tonaco Silva \\ ORCID: https://orcid.org/0000-0002-2589-3752 \\ Instituto Tocantinense Presidente Antônio Carlos, Brasil \\ E-mail: mariannatonaco-s@ hotmail.com \\ Ellen Lelis Souza \\ ORCID: https://orcid.org/0000-0003-2166-6103 \\ Instituto Tocantinense Presidente Antônio Carlos, Brasil \\ E-mail:ellenlelis@icloud.com \\ Marianne Morais de Pinho Fonseca \\ ORCID: https://orcid.org/ 0000-0002-8467-1789 \\ Instituto Tocantinense Presidente Antônio Carlos, Brasil \\ E-mail: mariannemorais80@gmail.com \\ Wanderson Stewart Parreira Miranda \\ ORCID: https://orcid.org/0000-0002-2228-541X \\ Instituto Tocantinense Presidente Antônio Carlos, Brasil \\ E-mail:stewart-2005@ hotmail.com \\ Rodrigo Ventura Rodrigues \\ ORCID: https://orcid.org/0000-0002-7312-6304 \\ Instituto Tocantinense Presidente Antônio Carlos, Brasil \\ E-mail: rodrigo.rodrigues@itpacpalmas.com.br
}

\begin{abstract}
Resumo
Objetivo: Investigar a aplicabilidade e os beneficios da terapia do canabidiol na melhora da qualidade de vida dos pacientes com doença de parkinson. Metodologia: Trata-se de uma revisão integrativa de literatura onde foram selecionados artigos utilizando as plataformas do BIREME, SCIENCE, EBSCO, PUBMED, LILACS e escolhidos para o espaço amostral da pesquisa apenas artigos completos em língua portuguesa, inglesa e alemão e publicados entre 1995 a 2020. Resultados: Diante dos fatos, o presente artigo trouxe a eficácia do canabidiol para o tratamento de primeira escolha para doença de Parkinson, bem como promover aos portadores e seus familiares um melhor convívio com a doença, e reflexos positivos em todos os âmbitos para uma melhor qualidade de vida. Conclusão: Embora o canabidiol tenha apresentado eficácia na terapia dos pacientes parkinsonianos, ainda se evidencia a necessidade de mais estudos e investigações em relação aos seus efeitos terapêuticos. Sendo assim, esse composto poderá se tornar o tratamento de primeira escolha para DP, e promover aos portadores e familiares um melhor convívio com a doença.

Palavras-chave: Canabidiol; Qualidade de vida; Doença de Parkinson; Usos terapêuticos.
\end{abstract}

\begin{abstract}
Objective: To investigate the applicability and benefits of cannabidiol therapy in improving the quality of life of patients with Parkinson's disease. Methodology: This is an integrative literature review where articles were selected using the platforms of BIREME, SCIENCE, EBSCO, PUBMED, LILACS and only complete articles in Portuguese, English and German and published among 1995 to 2020. Results: Given the facts, this article brought the effectiveness of cannabidiol for the treatment of first choice for Parkinson's disease, and to promote patients and their families a better coexistence with the disease, and positive effects in all areas for a better quality of life. Conclusion: Although cannabidiol has shown efficacy in the therapy of parkinsonian patients, the need for further studies and investigations regarding its therapeutic effects is still evident. Thus, cannabidiol could become the treatment of choice for PD, and promote better coexistence with the disease for patients and their families.
\end{abstract}

Keywords: Cannabidiol; Quality of life; Parkinson disease; Therapeutic uses.

\section{Resumen}

Objetivo: investigar la aplicabilidad y los beneficios de la terapia con cannabidiol en la mejora de la calidad de vida de los pacientes con enfermedad de Parkinson. Metodología: Se trata de una revision integradora de la literature donde se seleccionaron artículos utilizando las plataformas de BIREME, SCIENCE, EBSCO, PUBMED, LILACS y 
solo para el espacio muestral de la investigación se eligieron artículos completes en portugues, ingles y alemán y publicados entre 1995 y 2020. Resultados: Dados los hechos, este artículo trajo la efectividad del cannabidiol como tratamiento de primeira elección para la enfermedad de Parkinson, y para promover a los pacientes y sus familias una mejor convivencia con la enfermedad, y efectos positivos en todos los ámbitos para una mejor calidad de vida. Conclusión: Aunque el cannabidiol ha demonstrado su eficacia en la terapia de pacientes parkinsonianos, todavía es evidente la necessidad de realizar más estudios e investigaciones sobre sus efectos terapéuticos. Así, el cannabidiol podría convertirse en el tratamiento de elección para la DP y favorecer una mejor convivencia con la enfermedad de los pacientes y sus familiares.

Palabras clave: Cannabidiol; Calidad de vida; Enfermedad de Parkinson; Usos terapéuticos.

\section{Introdução}

A Doença de Parkinson (DP) é um processo neurodegerativo, causado por um desequilíbrio entre a ação inibitória da dopamina e a ação excitatória da acetilcolina devido à diminuição de dopamina (Rizec, 2016). A incidência e prevalência dessa doença aumentam com a idade atingindo as pessoas mais velhas e interferindo na qualidade de vida desses pacientes. A doença tem um curso progressivo e é caracterizada pela degeneração dos neurônios dopaminérgicos. Embora até a data não tenha sido encontrados tratamentos neuroprotetores para a DP, o sistema endocanabinoide surgiu como um alvo promissor (Chagas, 2014).

Os sintomas da doença de parkinson incluem alterações motoras como, rigidez, bradicinesia, tremor ao repouso, instabilidade postural, além de alterações não motoras como alterações cognitivas, autonômicas e depressão. No geral, é uma relação entre fatores genéticos e fatores ambientais. Foi observado ainda, no ano de 2019, estudos sobre o uso do cannabidiol (CBD) no tratamento da doença de Parkinson. Testes observacionais e clínicos realizados em pacientes Parkinsonianos mostraram efeitos positivos da cannabis relacionados aos comportamentos (Dos Santos, 2019).

Os pacientes com doença de parkinson ao serem submetidos ao uso do canabidiol para tratamento, podem apresentar resultados favoráveis, o que possibilita abordagem direcionada para os grupos mais susceptíveis com sintomas motores e cognitivos; podendo então, ser o ponto de partida para a priorização do desenho de medidas de eficácia e promover melhora na vida desses pacientes. Outrossim, os serviços de saúde devem adotar medidas preventivas ao uso do canabidiol, com o intuito de evitar a ocorrência de piora nos sintomas dos pacientes parkinsonianos. Surge a necessidade do devido conhecimento sobre o perfil dessas pessoas, possibilitando a identificação de pontos de eficácia para, assim, diminuir o número de sintomas motores, cognitivos e os pacientes obter uma melhor qualidade de vida (Shohet, 2017).

Dessa forma, faz- se necessário a pesquisa de novas alternativas terapêuticas visando melhorar o bem-estar dos pacientes portadores de doença de parkinson. O cannabidiol, através de estudos, tem se mostrado um composto promissor que pode refletir positivamente na vida desses pacientes por meio da sua eficácia, oferencendo assim, longevidade e melhora no bem estar geral.

O presente estudo tem como objetivo investigar a aplicabilidade e os beneficios da terapia com canabidiol na melhora da qualidade de vida dos pacientes com doença de parkinson. Trazendo a tona que, por mais que o canabidiol seja ilícito no Brasil na maior parte dos casos, de alguma forma está ligado à melhoria no bem-estar emocional e qualidade de vida desses pacientes.

\section{Metodologia}

Foi utilizado o método de pesquisa bibliográfica, por meio de uma revisão integrativa de literatura, que se trata da síntese de conhecimentos e reunião dos resultados de estudos relevantes que permite fundamentar a prática sobre determinado tema em conhecimentos científicos (Sousa, 2017). 
A seleção dos artigos para composição do espaço amostral se deu por meio de consulta de bases de dados eletrônicos Scientific ElectronicLibrary Online (SciELO), Biblioteca Regional de Medicina (BIREME), Sociedade para o Desenvolvimento da Pesquisa Científica (SCIENCE), Business Source Complete (EBSCO), National Library of Medicine (PUBMED) e Literatura Latino-americana do Caribe em Ciências da Saúde (LILACS). O trabalho foi realizado nos meses de setembro de 2020 à agosto de 2021.

O estudo pautou-se na seguinte questão de pesquisa: A eficácia do uso do Cannabidiol retrata melhoria na qualidade de vida dos pacientes com Parkinson?

Foi associado o descritor cannabidiol às palavras: usos terapêuticos, doença de parkinson e qualidade de vida. Os critérios de inclusão das referências foram definidos a inclusão apenas de artigos completos em língua portuguesa, inglesa e alemã pertencentes às bases de dados citadas acima e publicados entre 1995 a 2020. Foram excluídos os artigos pagos, não associados ao tema, que não comprovaram a eficácia da substância ao distúrbio neurodegenerativo e as duplicações de artigos.

Dessa maneira, foram abordadas fases para chegar à revisão integrativa como, identificar o problema, fazer uma busca na literatura mediante artigos, palavras-chave, bases de dados, critérios de inclusão e exclusão, avaliação de dados, análise de dados e, uma apresentação dos dados finais observando como estes estão relacionados na teoria.

Foram selecionados 23 artigos originais para o espaço amostral da pesquisa, e outras 16 revisões bibliográficas foram escolhidas para realizar a discussão. Foram excluídos outros 7 artigos por não tratarem sobre os benefícios do cannabidiol na melhora da qualidade de vida dos pacientes com doença de parkinson.

\section{Resultados e Discussão}

A Cannabis Sativa medicinal é utilizada há muitos anos, contudo existe um estigma por parte da sociedade e também por alguns governos, na qual proíbe tanto o uso na forma fumada até seu uso medicinal (Hannah, 2019). Devido a estudos atuais sabe-se que essa planta possui propriedades muito importantes que vão desde a proteção contra o estresse oxidativo nas células neurais à diminuição de sintomas motores provocadas por algumas doenças como a doença de Parkinson.

Em estudos pré-clínicos mais recentes em humanos o canabidiol mostrou-se promissor, pois sugerem que tanto as drogas antagonistas como as agonistas do receptor CB1 possuem potencial quanto ao uso medicinal do CBD na melhora das manifestações clínicas motoras da DP, devendo-se ao fato de elevados níveis de receptores CB1 e CB2 nos gânglios da base e cerebelo, regiões necessárias para os movimentos corporais. Os tratamentos atuais aprovados incluem a levodopa, que têm por mecanismo de ação básico a capacidade de ser convertida em dopamina dentro do cérebro e corrigir o estado de deficiência característico do Parkinsonismo. Mas, como outras drogas, ela apresenta muitos efeitos adversos como a intolerância gastrintestinal mais observado no início do tratamento e, além disso, alterações psiquiátricas e hipotensão ortostática e é uma droga dose dependente, ou seja, com o passar do tempo são necessárias doses maiores para atingir efeito terapêutico, contudo esse incremento de dose também aumenta os efeitos adversos (Rocha, 1995).

O cannabidiol é um composto não psicomimético da Cannabis Sativa que manifesta efeitos antipsicóticos, ansiolíticos, antiinflamatórios e neuroprotetores. Nos pacientes com parkinson, o CBD atua diminuindo os sintomas psicóticos induzidos por discinesia (LID) e com isso melhoria na sua qualidade de vida (Peres, Fernanda F, 2016). Contudo, com o avançar da doença as doses de levodopa devem ser reajustadas e podem acontecer fenômenos dos tipos, flutuação motora sendo este o mais comum, a "deterioração de final de dose" (wearing-off) e as flutuações aleatórias, também conhecidas como "fenômeno on-off. Com esse fenômeno o manejo da dose na qual o paciente deve tomar torna-se muito difícil, com isso ocorre piora da sua qualidade de vida (Rocha, 1995). Assim, um estudo publicado na Academia de Neurologia Brasileira cita que há evidências suficientes para indicar o uso dos derivados da cannabis em pacientes com distúrbios dos movimentos, quando os tratamentos atuais para estes são ineficazes ou intoleráveis (Bruck, 2015). 
Relata-se ainda que, existem sinais que afirmam que o uso do extrato da planta, em especial o CBD, ajude a minimizar sintomas não motores da DP como: psicose, distúrbios do sono, dor, talvez urgência miccional, e também promover melhora da qualidade de vida dos pacientes. A forma de uso deve ser CBD puro e extratos de cannabis com baixo teor de THC, pois eles parecem ser mais eficientes e menos propensos a causar efeitos colaterais indesejáveis (Bruck, 2015).

A qualidade de vida relacionada com a saúde é definida como "a percepção e avaliação pelos próprios pacientes do impacto causado na sua vida pela doença e as suas consequências". Além disso, pode-se citar a combinação de bem-estar físico, psicológico e social no contexto de uma doença em particular, e é uma forma de medir as condições de vida (Barone $\mathrm{P}$, 2017). Apesar disso, há a necessidade de mais ensaios clínicos plausíveis nos sintomas não motores. Tende a atuar na especialidade interdisciplinar e concentra como forma de prevenção, diminuição de sintomas, alívio e sofrimento nos pacientes com a doença de parkinson (David Oliver, 2020).

Em estudo do efeito da cannabis médica nas medições quantitativas térmicas da dor em pacientes com doença de parkinson, retratou nestes doentes que tiveram a autorização de usar a cannabis com o intuito de avaliar a função motora e a dor. Com isso, foram submetidos avaliação e questionários por "Classificação da Dor", "Escala Visual Analógica (VAS)", "Escala Unified PD Rating (UPDRS)" após o uso de cannabis. Após o resultado de questionários com pontuações da doença, foi observado uma melhoria nas funções motoras e os sintomas de dor dos pacientes com DP (Shohet, 2017). Assim, observase a relação de uma melhor qualidade de vida com diminuição da dor e funções motoras á esses pacientes com DP.

Visto que, o principal objetivo do estudo é comprovar a eficácia do canabidiol na melhoria da qualidade de vida dos pacientes portadores de doença de parkinson, foram selecionados 16 artigos dos quais 15 mostraram eficácia no uso do canabidiol, sendo que 1 artigo apresentado na tabela, discorreu acerca do uso da levodopa e relatou maiores efeitos adversos em comparação ao com uso do CBD. Além disso, foi observado que o canabidiol é bem tolerado e com menos efeitos adversos significativos.

Quadro 1: Eficácia pelo canabidiol com o devido efeito na melhoria do paciente com Parkinson.

\begin{tabular}{|l|l|l|}
\hline Autores & Eficácia do Canabidiol & Tipo de Melhoria em DP \\
\hline Chagas, 2014 & SIM & $\begin{array}{l}\text { Melhoria das medidas de qualidade de vida sem comorbidades } \\
\text { psiquiátricas. }\end{array}$ \\
\hline Dos Santos, 2019 & SIM & Melhoria nos sintomas motores. \\
\hline Poças, 2017 & SIM & $\begin{array}{l}\text { Melhoria Controle dos movimentos involuntários } \\
\text { Melhoria nos tremores } \\
\text { Melhoria da bradicinesia } \\
\text { Melhoria da LID. }\end{array}$ \\
\hline Filho, 2019 & SIM & Indução e estabilização dos sintomas causados pelo Parkinson. \\
\hline Sunaga, 2018 & SIM & $\begin{array}{l}\text { Atua em diferentes regiões corticais relacionados ao controle } \\
\text { motor, através da interação com os sistemas gabaérgicos, } \\
\text { glutamatérgicos e dopaminérgicos. }\end{array}$ \\
\hline Leehey, 2017 & SIM & Melhoria tremor, a ansiedade e a psicose. \\
\hline Crippa, 2010 & SIM & Melhoria da ansiedade e Sintomas psicóticos. \\
\hline Cripaa, 2010 & SIM & $\begin{array}{l}\text { Atividade ansiolítica- reduzindo a atividade, causando } \\
\text { sonolência e induzindo à calma. }\end{array}$ \\
\hline Pedrazzi, 2014 & SIM & Reduz euforia. \\
\hline Kindred, 2017 & SIM & SIM \\
\hline Balash, 2017 & SIM & $\begin{array}{l}\text { Resultados positivos em relação a alguns sintomas cognitivos; } \\
\text { os pacientes relataram diminuição das quedas, sensação de dor, } \\
\text { depressão e insônia }\end{array}$ \\
\hline Peres, Fernanda F, 2016 & $\begin{array}{l}\text { Diminuição os sintomas psicóticos induzidos por discinesia } \\
\text { (LID) e de melhorar a qualidade de vida. }\end{array}$ \\
\hline
\end{tabular}




\begin{tabular}{|l|l|l|}
\hline Barone P, 2017 & SIM & Melhoria bem-estar físico, psicológico e social. \\
\hline David Oliver, 2020 & SIM & Diminuição de sintomas, alívio e sofrimento. \\
\hline Shohet, 2017 & SIM & Melhoria nas funções motoras e os sintomas de dor. \\
\hline Rocha, 1995 & USO DO LEVEDOPA & $\begin{array}{l}\text { Efeitos adversos como vômitos, sudorese, hipotensão } \\
\text { ortostática, alucinações e discinesia (LID). }\end{array}$ \\
\hline
\end{tabular}

Fonte: Autores.

O Quadro 1, resume os detalhes dos 16 estudos científicos que investigaram a eficácia e o tipo de melhoria dos sintomas em doença de Parkinson. Com isso, Rocha et al., (1995) estudou 176 pacientes em uso de levedopa que apresentaram movimentos involuntários pelo uso do tratamento. Sendo 67 do sexo feminino e 109 do sexo masculino, com idade na DP de 13 a 80 anos com uma média de $52 \%$ e, uma média do tempo de uso da levedopa até o momento de aparecimento de discinesias de 4,2 anos. Outrossim, 85\% dos pacientes com discinesia com o uso de levedopa de dosagem de $800 \mathrm{mg}$ ao dia, sendo um total da média de dose ao dia de 614,4mg. Além disso, 104 pacientes apresentaram flutuações motores significativas junto à discinesia. Relacionando ao tipo de movimento, o coreiforme houve predominância em $34,7 \%$ dos casos retratados. Fica evidente que, por mais que a levedopa seja um fármaco de uso da atualidade no Brasil para tratar a doença de Parkinson, as discinesias são sintomas frequentes nos pacientes com Parkinson tratados com o uso do fármaco levedopa, sendo que varia a prevalência de $18 \%$ a $91 \%$ dessa queixa em diversas literaturas presentes nos pacientes de uso por mais de 5 anos de uso da levedopa. Em consideração a isso, acredita-se que dependendo do grau de comprometimento clínico dos pacientes, seja fator provocador para o aparecimento das discinesias e efeitos adversos.

Está documentado que a cannabis causa efeitos adversos. Em uma revisão sistemática e meta-análise de 69 estudos transversais de adolescentes, jovens e adultos com 2.152 usuários de cannabis e 6.575 participantes de comparação. Com isso, mostrou um pequeno tamanho do efeito geral para redução do funcionamento cognitivo em usuários frequentes de cannabis (d=-0,25; de IC de 95\% -0,32 a -0,17), 5 dos 8 domínios foram afeados como velocidade de processamento de informações, aprendizagem, memória atrasada, atenção, aspecto de função executiva. Disso, 15 estudos exigiram 72 horas ou mais de abstinência de cannabis, antes do teste não demostraram um efeito no funcionamento cognitivo (Uptodate, 2021). Assim, sugere que os efeitos da cannabis eram limitados no tempo.

Visto que o CBD é neuroprotetor, possivelmente com base em relatos de efeitos antioxidantes e anti-inflamatórios. Na confirmação desse estudo do regime de dosagem de CBD na forma de "GWP42003-P". A fabricação do medicamento recomendada pelo fabricante do estudo é segura e tolerável em 10 pacientes com doença de Parkinson. O “GWP42003-P” é iniciado com $5 \mathrm{mg} / \mathrm{kg} / \mathrm{dia}$ e aumentado em $5 \mathrm{mg} / \mathrm{kg}$ para uma dose alvo de $25 \mathrm{mg} / \mathrm{kg} / \mathrm{dia}$ em intervalos de 3 dias. Sendo assim, o CBD purificado é um líquido com sabor de morango em óleo de gergelim extraído como $100 \mathrm{mg} / \mathrm{ml}$ de material vegetal com alto teor de CBD. Com isso, o canabidiol mostra evidências que é seguro como neuro protetor, redução de tremores, além de limitar o efeito psicoativo do THC, com propriedades antiinflamatorias, anticonvulsivantes, antioxidantes, antipsicóticas e ansiolíticas (Leehey, 2017).

A comparação retrata uma redução dos sintomas na doença de Parkinson, levando a uma melhoria na qualidade de vida dos pacientes que utilizam o canabidiol. Posto isto, um componente da cannabis que se mostrou relativamente seguro e potencialmente neuroprotetor, reduz o tremor, a ansiedade, a psicose, e é bem tolerado na doença de parkinson. Devido a isso, o uso do CBD demonstra resultados favoráveis tanto em estudos pré-clínicos como em estudos clínicos.

\section{Conclusão}

Com base nos resultados dos estudos compreende-se que, a doença de parkinson é uma patologia que afeta inteiramente a qualidade de vida dos seus portadores, visto que, sua tendência é a progressão e o seu comprometimento é 
motor, cognitivo e autonômico, faz-se necessária a busca por outras alternativas terapêuticas a fim de poder proporcionar aos pacientes bem-estar geral e controle da sintomatologia que, com o tempo e efeitos adversos dos medicamentos tende a aumentar e prejudicar ainda mais a funcionalidade do indivíduo. Desse modo, os tratamentos atuais e legais para a doença de Parkinson até o momento são tidos como os mais efetivos, entretanto, estudos afirmaram que eles apesar de serem benéficos, também possuem uma parcela prejudicial, pois apresentam efeitos colaterais e ainda piora progressiva dos sintomas e não tem efeitos na progressão da doença. Além disso, com o passar do tempo as dosagens precisam ser aumentadas, pois perdem a aplicabilidade, restando dessa forma, somente os muitos efeitos colaterais. Assim, este trabalho teve como objetivo principal comprovar que o canabidiol, composto extraído da Cannabis Sativa mais popularmente conhecida como maconha, pode ser promissora em se tratando da melhora da qualidade de vida dos pacientes portadores de Doença de Parkinson no que se refere à sintomatologia motora e cognitiva.

Sendo assim, pode-se tão logo ser realizada pesquisas e testagens mais amplos, com o fito de melhor percepção, resposta, análise e conhecimento da atuação positiva do canabidiol sobre a doença de parkinson, a fim de poder lançá-lo como terapia eficaz no tratamento e controle da doença de Parkinson. Assim, supere as atuais terapias existentes e utilizadas, podendo assim promover um melhor convívio do portador com a sua doença e ofertar uma melhora do seu bem-estar e qualidade de vida.

\section{Agradecimentos}

Ao ITPAC, seu corpo docente, direção e administração que oportunizaram a janela de um horizonte superior. A nossa coordenadora da disciplina Lorena Dias Monteiro, por seu tempo, correções e incentivo do projeto. E a todos que direta ou indiretamente fizeram parte do projeto.

\section{Referências}

Aarsland D., Ballard C., Walker Z., Bostrom F., Alves G., Kossakowski K., Leroi I., Pozo-Rodriguez F., Minthon L. \& Londos E. (2009). Memantine in patients with Parkinson's disease dementia or dementia with Lewy bodies: a double-blind, placebo-controlled, multicentre trial. Lancet Neurol.

Balash Y, Bar-Lev Schleider L, Korczyn AD, Shabtai H, Knaani J, Rosenberg A, Baruch Y, Djaldetti R, Giladi N, Gurevich T. Medical Cannabis in Parkinson Disease: Real-Life Patients' Experience. Clin Neuropharmacol. 40(6):268-272. 10.1097/WNF.0000000000000246.

Barbosa, E. R. \& Sallem F. A. S. (2005). Doença de Parkinson. Revista neurociências, 13(3), 158-165.

Barone, P., Erro, R., \& Picillo, M. Quality of Life and Nonmotor Symptoms in Parkinson's Disease. Int Rev Neurobiol., 133:499-516

Bruck, S. (2015). Cannabinoids in neurology-Brazilian Academy of Neurology. Arquivos de neuro-psiquiatria, 73 (4), $371-374$.

Camargo Filho, M., Romanini, A., Pyrich, B., Pedri, E., Fontoura, G., Zorrer, L., Gonçalves, V., Gianini, V., \& Müller, J. (2019). Canabinoides como uma nova opção terapêutica nas doenças de Parkinson e de Alzheimer: uma revisão de literatura. Revista Brasileira de Neurologia, 55(2).

Campbell. (2014). W.W. DeJong: O Exame Neurológico. (7a ed.), Guanabara Koogan Ltda.

Chagas, M. H. N. (2014). Effects of cannabidiol in the treatment of patients with Parkinson's disease: an exploratory double-blind trial. Journal of Psychopharmacology, 28 (11), 1088-1098.

Leehey, Maureen Anne. A study of tolerability and Efficacy of Cannabidiol on Tremor in Parkinson's Disease. 2017. https://clinicaltrials.gov/ct2/show/study/NCT02818777.

Crippa, J. A.S., Zuardi, A. W., H., \& Jaime E. C. (2010). Uso terapêutico dos canabinoides em psiquiatria. Brazilian Journal of Psychiatry, 32 , $556-566$.

Deiana S, W. A, Yamasaki Y., Amada N., Arthur M., Fleming S., Woodcock H., Dorward P., Pigliacampo B., Close S., Platt B., \& Riedel G. (2012). Plasma and brain pharmacokinetic profile of cannabidiol (CBD), cannabidivarine (CBDV), $\Delta^{9}$-tetrahydrocannabivarin (THCV) and cannabigerol (CBG) in rats and mice following oral and intraperitoneal administration and CBD action on obsessive-compulsive behaviour. Psychopharmacology (Berl).

Dos Santos, R. G., Hallak, J. E. C \& Crippa, J. A. S. (2019). O uso do Canabidiol (CBD) no tratamento da Doença de Parkinson e suas comorbidades. Revista de Medicina, 98 (1), 46-51.

Gurgel, H. L. C. (2019). Uso terapêutico do canabidiol: a demanda judicial no estado de Pernambuco, Brasil. Saúde e Sociedade, $28,283-295$. 
Research, Society and Development, v. 10, n. 13, e09101320768, 2021

(CC BY 4.0) | ISSN 2525-3409 | DOI: http://dx.doi.org/10.33448/rsd-v10i13.20768

Iannott, F. A., Di M V. \& Petrosino S. (2016). Endocannabinoids and endocannabinoid-related mediators: targets, metabolism and role in neurological disorders. Progress in lipid research, 62, 107-128.

Katz M, G. Y. \& Kluger B. M. (2018). Top Ten Tips Palliative Care Clinicians Should Know About Parkinson's Disease and Related Disorders. J Palliat Med.

Kluger, B., Triolo, P., Jones W. \& Jankovic, J. - The Therapeutic Potential of Cannabinoids for Movement Disorders. HHS Public.

Martins, C. C. M., Caon, G., Moraes, \& Chaiane M. O. (2020). A Doença de Parkinson e o Processo de Envelhecimento Motor: uma Revisão de Literatura. Saúde e Desenvolvimento Humano, 8 (3) 155-167.

Mcpartland, John M. (2015). Are cannabidiol and $\Delta 9$ - tetrahydrocannabivarin negative modulators of the endocannabinoid system? A systematic review. British journal of pharmacology, 172 (3), 737-753.

Oliver, D. \& Veronese, S. (2020). Specialist palliative care for Parkinson's disease. Annals of Palliative Medicine. 2. 1201-1201. 10.21037/apm.2019.12.01.

Peres, F. F. (2016). Cannabidiol prevents motor and cognitive impairments induced by reserpine in rats. Frontiers in pharmacology, $7,343$.

Rocha, M. S. G. (2015). Discinesias induzidas por levodopa em 176 pacientes com doença de Parkinson. Arq. Neuro-Psiquiatr. 53 (4), 737-742.

Santos, E A. G. (2015). The neuroprotection of cannabidiol against MPP+-induced toxicity in PC12 cells involves trkA receptors, upregulation of axonal and synaptic proteins, neuritogenesis, and might be relevant to Parkinson's disease. Toxicology in Vitro, 30 (1), $231-240$.

Shohet, A. (2017). Effect of medical cannabis on thermal quantitative measurements of pain in patients with Parkinson's disease. European Journal of Pain, 21 (3), 486-493.

Souza, C. F. M. (2011). A Doença de Parkinson e o processo de envelhecimento motor. Revista Neurociências, 19 (4), $718-723$.

Sunaga, B. Y. (2018). Efeitos terapêuticos e tóxicos da Cannabis sativa. Trabalho de Conclusão de Curso (Graduação em Farmácia) - Universidade Federal de São Paulo - Campus Diadema.

Sousa, L. M. M. S., Marques-Vieira, C. M. A., Severino, S. S., \& Antunes, A. V. (2017). Metodologia de revisão integrativa da literatura em enfermagem.

University of Colorado, Denver. A Randomized, Double Blind, Placebo-controlled Crossover Study of Tolerability and Efficacy of Cannabidiol (CBD) on Tremor in Parkinson's Disease. Clinical trial registration, NCT02818777.

UpToDate. (2021). Cannabis use and disorder epidemiology comorbidity health consequences and medico legal status.

Woo, Y., \& Hyun, M. K. (2019). Effectiveness of Integrative Therapy for Parkinson's Disease Management. Frontiers in aging neuroscience, 11, 40. 